matics (e.g., set theory, geometry, Boolean algebra, measure theory), his principal contributions are along the lines of logic. The present volume contains his early logical work. (It does not, for instance, contain the Banach-Tarski paradox.) The seventeen papers that appear here in English translation, occasionally with slight changes, corrections, and additions, were first published in German, or French, or Polish. Since some of them were published in more than one part, or in more than one version, a complete list of the original sources would contain not seventeen but something more like thirty titles.

The longest paper is the famous Wahrheitsbegriff; it covers 127 pages. Since the first version of this work is in Polish, and the second (in German) appeared in a journal not readily available to mathematicians (Studia Philosophica), the reviewer, for one, would have rushed out to buy the book even if it contained nothing else. It does, however, contain a lot more. Here are a couple of tempting samples: the 1930 paper on the sentential calculus (with Lukasiewicz), and the 1933 paper on $\omega$-consistency and $\omega$-completeness.

The translation seems smooth. A noteworthy feature is the fact that, frequently, the notation was "translated" as well as the text; the letters (or digrams) used in this volume remind the reader, quite properly of course, of the English words "truth," "consequence," etc., rather than, say, the corresponding German words. The usability of the volume is further enhanced by a unified bibliography, a subject index, an index of names, and an index of symbols.

According to a letter from Tarski to the reviewer, Tarski's post1938 papers on the subject of this volume are all in English. Armed with this volume, therefore, and with the reviews in the Journal of Symbolic Logic, the English-speaking mathematical logician can be confident of having Tarski's complete logical output easily accessible to him-a circumstance for which many logicians should and will be grateful to the translator and publisher.

Paul R. Halmos

La theorie des fonctions de Bessel, exposée en vue de ses applications a la physique mathématique. By Gérard Petiau. Centre National de la Recherche Scientifique, Paris, 1955. 477 pp. 2500 francs.

This book is the first comprehensive treatise on Bessel functions since Watson's standard work on this subject. A comparison between the two is somewhat out of place since their purposes are different. The book under review, as its subtitle and its application part (more than 100 pages) indicate, is aimed to appeal mainly to the applied mathematician and physicist. Consequently those results and prop- 
erties which are likely to be of more importance with regard to applications than others are treated in more detail. Among the contributions since the appearance of Watson's book, mainly those with connection to applications are included. This of course is quite consistent with the purpose of this book. However, it seems regrettable to the reviewer that Chapter 5 (Asymptotic expansions) does not contain anything of the work done by R. E. Langer, T. M. Cherry, F. W. Olver and others. Likewise regrettable is the absence of a more detailed and organized bibliography. But these points may be of minor concern for the majority of the users of this book.

\section{F. OBERHETTINGER}

Symposium on Monte Carlo methods. Held at the University of Florida, March 16-17, 1954. Edited by H. A. Meyer. New York, Wiley, 1956. $16+382$ pp. $\$ 7.50$.

The term Monte Carlo methods was coined during the second World War to describe the use of random sampling for the numerical solution of mathematical problems. The questions that gave rise to these techniques had to do with the penetration of radiation into matter, in particular with the shielding properties important in the technology of atomic energy. Instead of solving the complicated integro-differential equations describing these processes by analytical or orthodox numerical methods it was proposed to simulate the physical phenomenon on computing machines by constructing artificially a sufficiently large number of particle biographies. Thus, in dealing with a process in which an elementary particle is subject to collisions resulting in absorption or reflection, possibly with a partial loss of energy, a moving particle with given initial energy and direction would be assumed and its subsequent history decided by playing a sequence of games of chance. The outcome of these games would decide from step to step the free path lengths, the nature of the collisions, the angles of reflection, the loss of energy in the collision, etc. The probability distributions underlying each game must, of course, be taken from physical theory. If a sufficient number of such fictitious biographies have been calculated, physically relevant questions such as the amount of radiation penetrating a given slab can be answered with reasonable accuracy.

The idea of solving mathematical problems by statistical experimentation is not new. Buffon's needle experiment for the determination of $\pi$ is well known, and statisticians have occasionally used such procedures to obtain preliminary information on the shape of distribution functions under mathematical investigation. However, these new 